\title{
ON AN INEQUALITY OF BOMBIERI
}

\author{
D. C. PEASLEE and W. A. COPPEL
}

(Received 24 August 1967)

Bieberbach's conjecture, proposed in 1916 and still unsolved, states that if $f(z)=z+a_{2} z^{2}+\cdots$ is holomorphic and univalent in the disc $|z|<1$ then $\left|a_{n}\right| \leqq n$ for each $n \geqq 2$, with equality for some $n$ only if $f(z)$ is the Koebe function

$$
k(z)=z /(1-z)^{2}=z+2 z^{2}+3 z^{3}+\cdots
$$

or is obtained from this function by a rotation. Very recently Bombieri has succeeded in showing that if $f(z)$ is sufficiently close to the Koebe function, then $\mathscr{R} a_{n} \leqq n$ with equality only if $f(z)=k(z)$. This had previously been proved by Garabedian, Ross and Schiffer [3] for even values of $n$.

In the announcement [1] Bombieri proves this result for functions $f_{\varepsilon}(z)$ depending analytically on a parameter $\varepsilon$ with $f_{0}(z)=k(z)$. The basis of the proof is the fact that the quadratic form

$$
R_{N}=\sum_{n=1}^{N-1} n(N-n) x_{n}^{2}+2 \sum_{m+n<N}(N-m-n) x_{m} x_{n}
$$

is positive definite, and Bombieri deduces this from the new integral inequality

$$
\int_{-1}^{1}\left(1-x^{2}\right) f^{2}(x) d x+2 \iint_{x+y \geqq 0}(x+y) f(x) f(y) d x d y \geqq 0,
$$

valid for all $f(x) \in L^{2}(-1,1)$. In the present paper a different proof of the inequality (2) is given, using Legendre polynomials. Moreover it is shown that equality holds only if $f(x)=\mathbf{0}$ almost everywhere. The positive definiteness of the quadratic form $R_{N}$ is also proved directly, without recourse to integrals, by using polynomials which are orthogonal over a finite set.

THEOREM. If $f(x) \in L^{2}(-1,1)$ and $0 \leqq \lambda \leqq 2$, then

$$
J(\lambda)=\int_{-1}^{1}\left(1-x^{2}\right) f^{2}(x) d x+\lambda \iint_{x+y \geqq 0}(x+y) f(x) f(y) d x d y \geqq 0,
$$

with equality only if $f(x)=0$ almost everywhere. If $\lambda<0$ or $\lambda>2$ there exist functions $f(x) \in L^{2}(-1,1)$ for which $J(\lambda)<0$.

Put

$$
F(x)=-\int_{399}^{1} f(\xi) d \xi
$$


Since $F(x)$ is absolutely continuous, it has a convergent expansion

where

$$
F(x)=\sum_{n=0}^{\infty} a_{n} \bar{P}_{n}(x)
$$

$$
\bar{P}_{n}(x)=\left(\frac{2 n+1}{2}\right)^{\frac{1}{2}} P_{n}(x)
$$

is the normalised Legendre polynomial and

$$
a_{n}=\int_{-1}^{1} F(x) \bar{P}_{n}(x) d x .
$$

By integrating by parts and using the differential equation for the Legendre polynomials we obtain

$$
\int_{-1}^{1}\left(1-x^{2}\right) f(x) \bar{P}_{n}^{\prime}(x) d x=n(n+1) a_{n} .
$$

In particular,

$$
\int_{-1}^{1}\left(1-x^{2}\right) \bar{P}_{m}^{\prime}(x) \bar{P}_{n}^{\prime}(x) d x=n(n+1) \delta_{m n} .
$$

Thus the polynomials $\bar{P}_{n}^{\prime}(x)(n \geqq 1)$ form an orthogonal system with respect to the weight function $w(x)=1-x^{2}$. Since the interval is finite, this system is complete. Therefore we have the Parseval relation

$$
J_{1} \equiv \int_{-1}^{1}\left(1-x^{2}\right) f^{2}(x) d x=\sum_{n=1}^{\infty} n(n+1) a_{n}^{2} .
$$

On the other hand, by integrating by parts we get

$$
\begin{aligned}
J_{2} & =\iint_{x+y \geq 0}(x+y) f(x) f(y) d x d y \\
& =\int_{-1}^{1} x f(x) \int_{-x}^{1} f(y) d y d x+\int_{-1}^{1} f(x) \int_{-x}^{1} y f(y) d y d x \\
& =-\int_{-1}^{1} x f(x) F(-x) d x+\left.F(x) \int_{-x}^{1} y f(y) d y\right|_{-1} ^{1}+\int_{-1}^{1} F(x) x f(-x) d x \\
& =\int_{-1}^{1} x[F(x) f(-x)-f(x) F(-x)] d x \\
& =-\int_{-1}^{1} x[F(x) F(-x)]^{\prime} d x \\
& =\int_{-1}^{1} F(x) F(-x) d x,
\end{aligned}
$$

since $F(1)=0$. Since $\bar{P}_{n}(-x)=(-1)^{n} \bar{P}_{n}(x)$ it follows from the Parseval relation for the system of Legendre polynomials that

$$
J_{2}=\sum_{n=0}^{\infty}(-1)^{n} a_{n}^{2}
$$


Thus

$$
\begin{aligned}
J(\lambda) & =J_{1}+\lambda J_{2} \\
& =\lambda a_{0}^{\mathbf{2}}+\sum_{n=1}^{\infty}\left[n(n+1)+\lambda(-1)^{n}\right] a_{n}^{\mathbf{2}} .
\end{aligned}
$$

This shows that $J(\lambda) \geqq 0$ if $0 \leqq \lambda \leqq 2$. Moreover if $J(\lambda)=0$ and $0 \leqq \lambda<2$ then $a_{n}=0(n \geqq 1)$ and hence $f(x)=0$ a.e. If $J(\lambda)=0$ and $\lambda=2$ then $a_{0}=0$ and $a_{n}=0(n \geqq 2)$. The vanishing of $a_{n}$ for $n \geqq 2$ implies that $f(x)$ is constant a.e. Since

$$
\begin{aligned}
2^{\frac{1}{2}} a_{0} & =\int_{-1}^{1} F(x) d x \\
& =-\int_{-1}^{1} f(x) d x-\int_{-1}^{1} x f(x) d x \\
& =-\int_{-1}^{1}(1+x) f(x) d x,
\end{aligned}
$$

this constant must be 0 .

Now write

$$
h_{N}=\sum_{n=1}^{N} \frac{2 n+1}{n(n+1)} \approx 2 \log N \quad \text { for } N \rightarrow \infty
$$

and let $f(x)$ be the polynomial defined by taking

$$
a_{n}= \begin{cases}(2 n+1)^{\frac{1}{2}} / n(n+1) & \text { for } 1 \leqq n \leqq N, \\ 0 & \text { for } n>N .\end{cases}
$$

Since $F(1)=0$ we have

Hence

$$
a_{0}=-\sum_{n=1}^{N}(2 n+1)^{\frac{1}{2}} a_{n}=-h_{N} .
$$

$$
J(\lambda)=h_{N}+\lambda\left[h_{N}^{2}+O(1)\right] .
$$

If $\lambda<0$ it follows that $J(\lambda)<0$ for sufficiently large $N$. On the other hand, if we define $a_{n}$ in the same way as before for $n \geqq 2$ but take

then $a_{0}=0$ and

$$
a_{1}=-3^{-\frac{1}{2}}\left(h_{N}-\frac{3}{2}\right) \text {, }
$$

$$
\begin{aligned}
J(\lambda) & =\frac{2}{3}\left(h_{N}-\frac{3}{2}\right)^{2}+h_{N}-\frac{3}{2}+\lambda \sum_{n=1}^{N}(-1)^{n} a_{n}^{2} \\
& =\frac{1}{3}(2-\lambda)\left(h_{N}-\frac{3}{2}\right)^{2}+h_{N}-\frac{3}{2}+\lambda O(1) .
\end{aligned}
$$

If $\lambda>2$ it follows that $J(\lambda)<0$ for sufficiently large $N$. This completes the proof of the theorem.

To prove the positive definiteness of the quadratic form $R_{N}$ directly, without using the inequality (2), we need the analogues of the Legendre polynomials for a discrete variable. Cebyšev has defined, for each positive 
integer $N$, a finite sequence of polynomials $t_{n}(x)(n=0,1, \cdots, N-1)$ with the following properties (see [2]):

(i) $t_{n}(x)$ has degree $n$ in $x$,

(ii) Orthogonality

$$
\sum_{x=0}^{N-1} t_{m}(x) t_{n}(x)=\beta_{n} \delta_{m n}
$$

where

$$
\beta_{n}=N\left(N^{2}-1^{2}\right)\left(N^{2}-2^{2}\right) \cdots\left(N^{2}-n^{2}\right) /(2 n+1),
$$

(iii) Symmetry

$$
t_{n}(N-1-x)=(-1)^{n} t_{n}(x),
$$

(iv) Difference equation

$$
\Delta\left[x(x-N) \Delta t_{n}(x-1)\right]-n(n+1) t_{n}(x)=0 .
$$

We will also require an orthogonality property of the first differences $\Delta t_{n}(x)$. By partial summation we get for $m, n=1, \cdots, N-1$

$$
\begin{aligned}
\sum_{x=0}^{N-1} x(x-N) \Delta t_{m}(x-1) \Delta t_{n}(x-1) & =-\sum_{x=0}^{N-1} t_{m}(x) \Delta\left[x(x-N) \Delta t_{n}(x-1)\right] \\
& =-n(n+1) \sum_{x=0}^{N-1} t_{m}(x) t_{n}(x) \\
& =-n(n+1) \beta_{n} \delta_{m n} .
\end{aligned}
$$

Let $f(x)$ be the uniquely determined polynomial of degree $<N-1$ such that $f(n-1)=x_{n}(n=1, \cdots, N-1)$. There exists a unique polynomial $F(x)$ of degree $<N$ such that $F(0)=0$ and

We can write

$$
f(x)=\Delta F(x)=F(x+1)-F(x) .
$$

$$
F(x)=\sum_{n=0}^{N-1} a_{n} \beta_{n}^{-\frac{1}{2}} t_{n}(x)
$$

with suitable coefficients $a_{n}$. Then

$$
f(x)=\sum_{n=1}^{N-1} a_{n} \beta_{n}^{-\frac{1}{2}} \Delta t_{n}(x) .
$$

We wish to evaluate the sum

$$
\begin{aligned}
S(\lambda) & =S_{1}+\lambda S_{2} \\
& =\sum_{x=1}^{N-1} x(N-x) f^{2}(x-1)+\lambda \sum_{\substack{x+y<N \\
x, y>0}}(N-x-y) f(x-1) f(y-1) .
\end{aligned}
$$


By (8) and (6)

$$
\begin{aligned}
S_{1} & =\sum_{m, n=1}^{N-1} a_{m} a_{n} \beta_{m}^{-\frac{1}{2}} \beta_{n}^{-\frac{1}{2}} \sum_{x=1}^{N-1} x(N-x) \Delta t_{m}(x-1) \Delta t_{n}(x-1) \\
& =\sum_{n=1}^{N-1} n(n+1) a_{n}^{2} .
\end{aligned}
$$

By the definition of $F(x)$ and by partial summation

$$
\begin{aligned}
S_{2} & =\sum_{x=1}^{N-2}(N-x) f(x-1) \sum_{y=1}^{N-1-x} f(y-1)-\sum_{x=1}^{N-2} f(x-1) \sum_{y=1}^{N-1-x} y f(y-1) \\
& =\sum_{x=1}^{N-2}(N-x) f(x-1) F(N-1-x)-\sum_{x=1}^{N-2}(N-1-x) f(N-2-x) F(x) \\
& =\sum_{x=0}^{N-2}(N-1-x)[f(x) F(N-2-x)-f(N-2-x) F(x)] \\
& =\sum_{x=1}^{N-1} x[f(N-1-x) F(x-1)-f(x-1) F(N-1-x)] \\
& =-\sum_{x=1}^{N-1} x \Delta[F(x-1) F(N-x)] \\
& =\sum_{x=0}^{N-1} F(x) F(N-1-x) .
\end{aligned}
$$

Therefore, by the symmetry property (4) and the orthogonality property (3),

$$
S_{2}=\sum_{n=0}^{N-1}(-1)^{n} a_{n}^{2}
$$

Thus

$$
S(\lambda)=\lambda a_{0}^{2}+\sum_{n=1}^{N-1}\left[n(n+1)+\lambda(-1)^{n}\right] a_{n}^{2} .
$$

Hence $S(\lambda) \geqq 0$ if $0<\lambda \leqq 2$, with equality only when $a_{0}=0$ and $a_{n}=0(2 \leqq n \leqq N-1)$. The vanishing of $a_{n}$ for $n \geqq 2$ implies that $f(x)$ is a constant. Since

$$
\begin{aligned}
a_{0} & =N^{-\frac{1}{2}} \sum_{x=0}^{N-1} F(x) \\
& =N^{-\frac{1}{2}} \sum_{x=0}^{N-1}(N-1-x) f(x)
\end{aligned}
$$

it follows that $f(x) \equiv 0$. In particular, for $\lambda=2$, this shows that the quadratic form $R_{N}$ is positive definite. 
[Added in proof: The complete proof of Bombieri's contribution to the Bieberbach conjecture has now appeared in Inventiones Math. 4 (1967), $26-27$.]

\section{References}

[1] E. Bombieri, 'Sulla seconda variazione della funzione di Koebe', Boll. Un. Mat. Ital. 22 (1967), 25-32.

[2] A. Erdélyi, et al., Higher transcendental functions (McGraw-Hill, New York, 1953), Vol. 2, p. 223.

[3] P. R. Grarabedian, G. G. Ross and M. Schiffer, 'On the Bieberbach conjecture for even $n$ ', J. Math. Mech. 14 (1965), 975-989.

Departments of Theoretical Physics and Mathematics

Institute of Advanced Studies

The Australian National University

Canberra, A.C.T. 